\title{
Plastics Pyrolysis and Two-stage Coliquefaction of Coal-plastic Mixtures
}

\author{
Kun $\mathrm{Du}^{\mathrm{a}^{*}}, \mathrm{Yu} \mathrm{Li}^{\mathrm{b}}$ and Minglei Lian ${ }^{\mathrm{c}}$ \\ Department of Chemistry and Chemical Engineering, Liu Pan Shui Normal University, Liupanshui, \\ Guizhou, China \\ a kundu408@163.com, b wangjin-tianya89@sohu.com, ${ }^{c}$ lingjueding579@tom.com \\ *Corresponding author Email:kundu408@gmail.com; kundu408@163.com
}

Keywords: Plastics pyrolysis, two-stage co-liquefaction, Medium-density polyethylene, Waste plastic Abstract. The two-stage co-processing of coal with medium-density polyethylene (MDPE) was investigated using ammonium tetrathiomolybdate (ATTM) as a catalyst. The first-stage plastic pyrolysis carried out at $420^{\circ} \mathrm{C}, 6.0 \mathrm{MPa}$ hydrogen pressure and $\mathrm{HZSM}-5$ as catalyst. The second-stage coal and MDPE co-liquefaction had been performed in a hydroprocessing unit at $430{ }^{\circ} \mathrm{C}$ and $6.0 \mathrm{MPa}$ hydrogen pressure with ATTM catalyst. A competitive experiment was performed by the way of one stage co-liquefaction of coal with MDPE using ATTM as catalyst and tetraline as solvent. The aim of the experiments was to determine the effect of the use of the waste plastic pyrolysis product as solvent. The results indicate that the hydroprocessed liquids of both the one stage and the two-stage co-processing of coal with MDPE have about $70 \%$ of compounds with boiling point below $350{ }^{\circ} \mathrm{C}$, and meet the sulphur and nitrogen specifications for refinery feedstocks.

\section{Introduction}

In recent years, disposal of plastic wastes has become an increasingly serious environmental concern. Due to an ever increasing volume of plastic products, sanitary landfills can no longer continue to be the major means for plastic wastes disposal. Plastics recycling appears to be a possible alternative solution and has potential for high growth. In order to obtain distillate liquids or petroleum compatible refined products from coal, addition of hydrogen is necessary ${ }^{[1-3]}$.

A possible method for hydrogen addition is co-processing of coal with polymeric waste materials since these latter materials contain hydrogen at levels much higher than are found in coal. The breakdown of waste rubber tires is interesting because the liquids derived may prove to be important as a coal dissolution and/or hydrogen donor solvent. Previous study shows that a solid acid catalyst (HZSM-5 zeolite) was highly active for the liquefaction of the plastics alone, typically giving oil yields of $80-95 \%$ and total conversions of $90-100 \%$ at temperatures of $430-450{ }^{\circ} \mathrm{C}^{[4-7]}$.

Two-stage coal liquefaction offers the potential for significant improvement over single-stage processing ${ }^{[8-10]}$. The objective of this research was to determine the feasibility of using two-stage co-processing for coal and MDPE reaction systems. The plastics (mainly polyethylene and polystyrene) were thermal cracked to obtain liquid products according to the different weight ratio of polyethylene and polystyrene. The liquid products were used as solvent for the second stage of co-liquefaction of Xingyuan coal and MDPE. Investigated the influence of the solvent which produced from the first stage by different weight ratio of polyethylene and polystyrene on the second stage of co-liquefaction of Xingyuan coal and MDPE. The results illustrate that there is an optimal weight ratio of polyethylene and polystyrene for the co-liquefaction of Xingyuan coal and MDPE.

\section{Experiment Section}

\section{Materials}

The sample of Xingyuan coal obtained from Guizhou Xinsheng Coal Chemical Co., Ltd., and dried sample of Xingyuan coal was ground to $<100$ mesh particles and used as a coal source. Table 1 lists the proximate and ultimate analyses of the coal. The proximate and ultimate analyses were all based on air dried basis. 
Table 1. Proximate and ultimate analyses (wt \%) of Xingyuan coal ${ }^{a}$

\begin{tabular}{|c|c|c|c|c|c|c|c|}
\hline \multicolumn{3}{|c|}{ Proximate analysis } & \multicolumn{5}{|c|}{ Ultimate analysis (daf) } \\
\hline $\mathrm{M}_{\mathrm{ad}}$ & $\mathrm{A}_{\mathrm{d}}$ & $\mathrm{V}_{\text {daf }}$ & $\mathrm{C}$ & $\mathrm{H}$ & $\mathrm{N}$ & $\mathrm{O}_{\text {diff }}$ & $\mathrm{S}_{\mathrm{t} . \mathrm{d}}$ \\
\hline 2.862 & 19.16 & 26.37 & 71.59 & 2.36 & 0.68 & 0.22 & 8.17 \\
\hline \multicolumn{8}{|c|}{${ }^{a}$ diff, by difference; daf, dry and ash-free basis; $\mathrm{M}_{\mathrm{ad}}$ moisture (air-dried basis); } \\
\hline \multicolumn{8}{|c|}{$A_{d}$, ash (dry basis); $V_{\text {daf }}$ volatile (dry and ash-free basis); and $S_{t . d}$, total sulfur ( dry basis) } \\
\hline
\end{tabular}

Polyethylene and polystyrene were supplied as $3.0 \mathrm{~mm}$ extruded pellets from Dongwan plastic Ltd. HZSM-5 and ATTM were purchased from Shanghai Shentan environmental protection new materials co., LTD. Pentane and tetrahydrofuran were purchased from Xiya reagent co., LTD.

\section{Preparation of mixed solvent with different weight ratio of polyethylene and polystyrene} In the first stage reaction, the different weight ratio of polyethylene and polystyrene mixtures were liquefied in order to obtain a liquid product to be used as the solvent in the second stage co-processing reaction. Fixed the weight of polyethylene at $200 \mathrm{~g}$, weighted $100 \mathrm{~g}, 200 \mathrm{~g}, 300 \mathrm{~g}, 400 \mathrm{~g}$ and $600 \mathrm{~g}$ polystyrene, respectively. To make the weight ratio of polyethylene and polystyrene at $2: 1,1: 1,1: 1.5$, $1: 2$ and $1: 3$.

The plastics mixtures were liquefied in a $1 \mathrm{~L}$ magneti-cally stirred autoclave at $420{ }^{\circ} \mathrm{C}$ for $120 \mathrm{~min}$ under an initial $\mathrm{H}$ pressure of 6.0 $\mathrm{MPa}$ introduced at ambient temperature. The reactors were agitated vertically at $450 \mathrm{rpm}$.

The loading for the HZSM-5 catalysts was 5 wt. \% on a plastics charge basis. After the reaction was completed, the reactor was quenched in ambient water. The liquid products were extracted with THF and then hexane, and the soluble amounts of each fraction were determined. The hexane solvent was evaporated from the hexane soluble liquid product, which was then used as the solvent for coal liquefaction in the second stage reaction.

\section{Co-liquefaction of Xingyuan coal and MDPE using the liquid product of pyrolysis as solvent}

The co-liquefaction experiments were conducted in 1L magneti-cally stirred autoclave which was shaken at $400 \mathrm{rpm}$ at the desired temperature. Usually 1:1 weight ratio of MDPE + Xingyuan coal, five times of solvent (based on the total weight of MDPE and Xingyuan coal, the liquid product of pyrolysis which produced in the first stage), $5 \%$ weight ratio of ATTM (based on the total weight of MDPE and Xingyuan coal) were charged in the $1 \mathrm{~L}$ magneti-cally stirred autoclave. The reaction times were 120 min. at $430{ }^{\circ} \mathrm{C}$, and the atmosphere in the magneti-cally stirred autoclave was $6.0 \mathrm{Mpa}$ cold $\mathrm{H}$ pressure.

After the reaction was over, the products were removed from the reactor with tetrahydrofuran (THF) and extracted in a Soxhlet apparatus. The THF solubles were subsequently separated into pentane soluble (Oil) and pentane insoluble (AS + PS) fractions. Total THF conversion was determined from the amount of insoluble material that remained (residue). Any added catalyst was subtracted from the residue sample weight.

There are totally 5 experiments, and they are designed as Two stage 1, Two stage 2, Two stage 3, Two stage 4 and Two stage 5, respectively. 


\section{Co-liquefaction of Xingyuan coal and MDPE using tetraline as solvent}

1:1 weight ratio of MDPE + Xingyuan coal, five times of tetraline solvent (based on the total weight of MDPE and Xingyuan coal), 5\% weight ratio of ATTM (based on the total weight of MDPE and Xingyuan coal) were charged in a $1 \mathrm{~L}$ magneti-cally stirred autoclave. The autoclave was heated to $430{ }^{\circ} \mathrm{C}$ under 6.0 Mpa initial $\mathrm{H}_{2}$ pressure, and maintained for 120 min under stirring at $500 \mathrm{RPM}$.

The products were divided into hexane soluble fraction as oil, the hexane insoluble-THF soluble fraction as asphaltene and preasphaltene (AS + PS), and THF insoluble fraction as residue. The experiment was designed as One stage 1.

\section{Molecular weight studies}

The $\mathrm{M}_{\mathrm{w}}$ and $\mathrm{M}_{\mathrm{n}}$ of oil and AS + PS obtained from Two stage 1, Two stage 3 and One stage 1 were measured in a UltiMate 3000 apparatus. Defining dispersivity (D) is $M_{w} / M_{n}$. D is an index for the homogeneity of the sample. All molecules of the sample have the same size when $\mathrm{D}=1.0$.

\section{Results and Discussion}

\section{Oil and total conversion of co-liquefaction of coal and MDPE in each experiment}

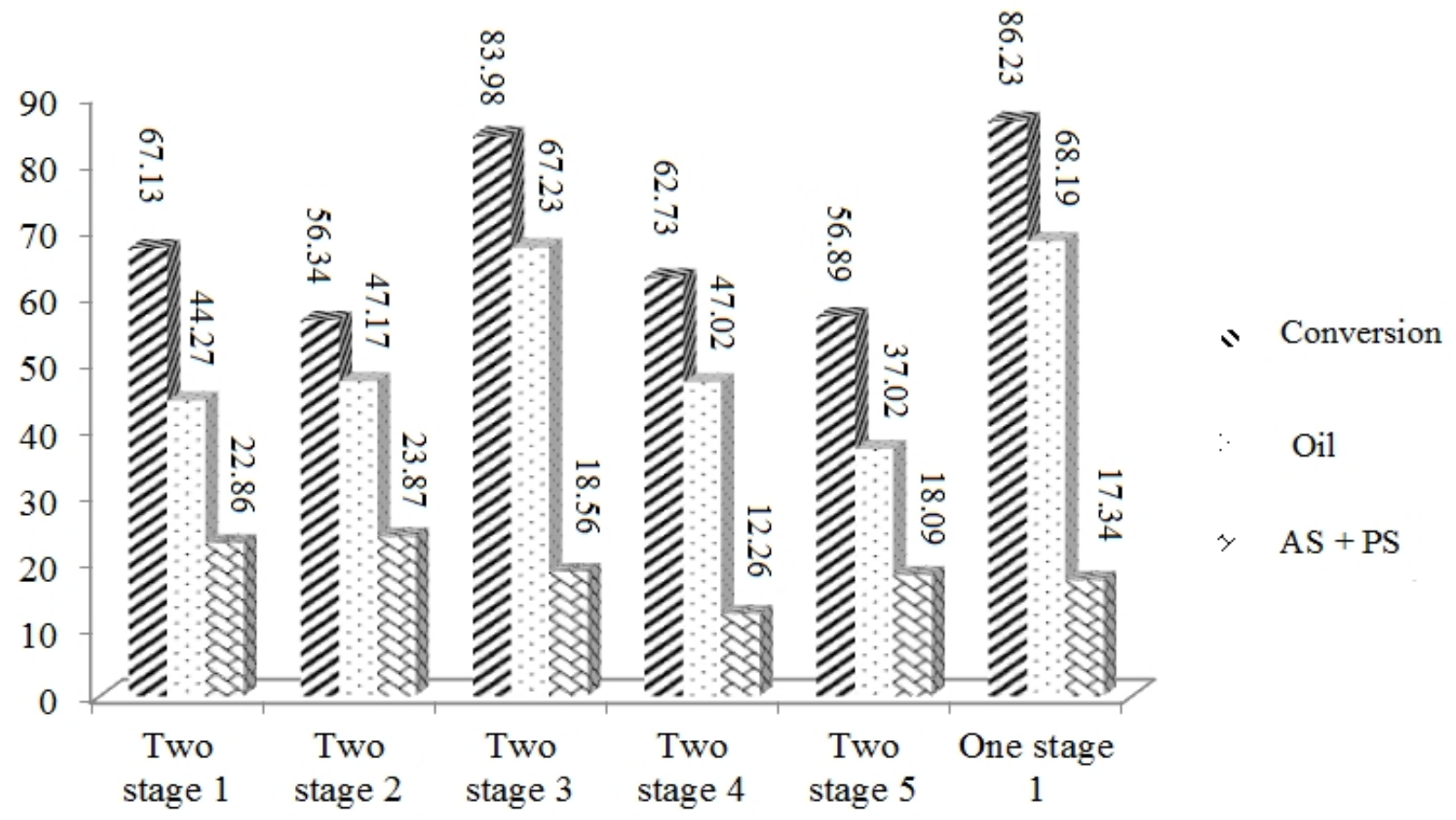

Figure 1. The oil and total conversion of co-liquefaction of Xingyuan coal and MDPE in each experiment.

Figure 1 shows the oil and total conversion of co-liquefaction of coal and MDPE in each experiment. As for the two stage co-liquefaction experiments, the co-liquefaction of coal and waster plastic needs the kind of solvent that either consists of aliphatic hydrocarbon or aromatic hydrocarbon. The authors prepared mixed solvent which contains aliphatic hydrocarbon structure and aromatic hydrocarbon structure. The aromatic hydrocarbon plays the role of loading $\mathrm{H}$ atom at the reaction of co-liquefaction of coal and waster plastic. These new hydrogenizes aromatic hydrocarbon speeds the $\mathrm{H}$ atom transmission of coal pyrolysis, thus makes the total conversion of coal liquefaction improved greatly.

The 1:1.5 weight ratio of polyethylene and polystyrene as solvent achieves the highest oil yield for 1:1 weight ratio of MDPE and Xingyuan coal co-liquefaction.

It also can be seen from One stage 1 that when a strong $\mathrm{H}$ donor such as tetraline as solvent was used at co-liquefaction of coal and waste plastic, it gets high oil yield and total coal conversion. It evinces 
that high active hydrogen density is important for the co-liquefaction.

\section{Molecular weight distribution}

The calculate results of the mass distribution of three products of liquefaction fractionated into oil, asphaltene and preasphaltene for Two stage 1, Two stage 3 and One stage 1 is shown in Table 2.

Table 2. Molecular weight data for liquid products obtained at $430{ }^{\circ} \mathrm{C}$

\begin{tabular}{cccc}
\hline & $\mathrm{M}_{\mathrm{n}}$ & $\mathrm{M}_{\mathrm{w}}$ & Dispersivity \\
\hline Two stage 1 & 370 & Hexane soluble (Oil) & 1.956 \\
Two stage 3 & 300 & 724 & 1.433 \\
One stage 1 & 278 & 430 & 1.366 \\
& Hexane insoluble-THF soluble (AS + PS) \\
Two stage 1 & 480 & 997 & 2.07 \\
Two stage 3 & 527 & 973 & 1.846 \\
One stage 1 & 420 & 719 & 1.711 \\
& \multicolumn{4}{c}{ Overall molecular weight data ${ }^{\mathrm{a}}$} \\
Two stage 1 & 273.5 & 548.4 & 2.005 \\
Two stage 3 & 299.5 & 469.6 & 1.568 \\
One stage 1 & 262.3 & 383.7 & 1.462 \\
\hline Determined from data for individual fractions and the mass fraction data of Figure 1.
\end{tabular}

It is obviously that lower amounts of hexane insoluble-THF soluble compounds and higher quantities of the desirable lower molecular weight Hexane soluble fractions over the corresponding fraction of liquids that are obtained by processing either Two stage 3 or One stage 1. Similar enhancement in coal conversion and improvement in product quality were observed in the two experiments. The results indicates that Two stage co-liquefaction of waste plastic and coal using the liquid product of pyrolysis as solvent in the second stage results in the lowering of $\mathrm{C} / \mathrm{H}$ ratios of the liquids obtained.

Whereas, the main reason for the mild condition of co-liquefaction of waste plastic and coal was the high $\mathrm{H}$ contribution of solvent. The paper prepared different ratio of solvent with aliphatic hydrocarbon and aromatic hydrocarbon to meet with the pyrolysis section of waste plastic and coal, thus synergetic effect of waste plastic and coal is happened, and the yield of oil improved. For co-liquefaction of MDPE and Xingyuan coal with 1:1 weight ratio, it is obviously that the best solvent which produced in the first stage is the 1:1.5 weight ratio of polyethylene and polystyrene, as compared to the rest four experiments in 2.2. Preparation of mixed solvent with different weight ratio of polyethylene and polystyrene

\section{Conclusions}

1. Exploratory reaction studies have shown two-stage coal co-processing with waste plasticss to yield high conversions of coal $(>80 \%)$ with a high selectivity for oil $(>60 \%)$. the different ratio of aliphatic hydrocarbon and aromatic hydrocarbon were first thermally cracked in a first stage to yield a liquid solvent for the second-stage coal and MDPE co-processing.

2. New results for plastics liquefaction and coal-plastic co-liquefaction suggest that the nature of the plastic, the solvent, and the reaction atmosphere can all have a significant on product yields. A thorough experimental matrix is needed to explore this parameter space. Further catalyst 
development aimed at producing cheaper, more robust catalysts for coal-waste plastic co-liquefaction is required.

\section{Acknowledgements}

This work was performed with the financial assistance of the Guizhou Provincial Department of Education 2011 Key Research Projects (NO: [2011]232) and Guizhou Provincial Department of S \& T 2011 Projects of Social Development through the Research Project (NO: 20113112).

\section{References}

[1] J. Yanik, M. A. Uddin, Y. Sakata, The effect of red mud on the liquefaction of waste plastics in heavy vacuum gas oil, Energy Fuels. 15 (2001) 163-169.

[2] P. Sivakumar, H. Jung, J. W. Tierney, I. Wender, Liquefaction of lignocellulosic and with coal using carbon monoxide alkali plastic wastes and aqueous, Fuel Process. Technol. 49 (1996) 219-232.

[3] H. K. Joo, C. W. Curtis, Catalytic coprocessing of LDPE with coal and petroleum resid using different catalysts, Fuel Process. Technol. 53 (1998) 197-214.

[4] C. Song, A. K. Sainit, Strong synergistic effect between dispersed Mo catalyst and $\mathrm{H}_{2} \mathrm{O}$ for low-severity coal hydroliquefaction, Energy Fuels. 9 (1995) 188-189.

[5] L.Wang, P. Chen, Mechanism study of iron-based catslysts in co-liquefaction of coal with waste plastics, Fuel. 81 (2002) 811-815.

[6] A.G. Buekens, H. Huang, Catalytic plastics cracking for recovery of gasoline-range hydrocarbons from municipal plastic wastes, Resour. Conserv. Recycl. 23 (1998) 163-181.

[7] A. López, I. d. Marco, B.M. Caballero, M.F. Laresgoiti, A. Adrados, Pyrolysis of municipal plastic wastes: Influence of raw material composition, Waste Manage. 30 (2010) 620-627.

[8] W. Ding, J. Liang, L. L. Anderson, Two-stage co-processing of waste plastics with coal, ACS.Div.Fuel Chem. 41(1996) 1037-1042.

[9] H. K. Joo, J. N. Hool, C. W. Curtis, ANOVA analysis of two-stage coprocessing of low-density polyethylene, coal, and petroleum resid, Energy Fuels. 12 (1998) 704-714.

[10] C. W. Curtis, J. A. Guin, A. R. Tarrer, W. Huang, Two-stage coal liquefaction using sequential mineral and hydrotreating catalysts, Fuel Process. Technol. 7 (1983) 277-291. 